\title{
Uso da técnica de confusão sexual no manejo populacional de Cryptoblabes gnidiella (Lepidoptera: Pyralidae) em videira
}

\author{
José Eudes de Morais Oliveira( ${ }^{(1)}$, Maria Herlândia de Araújo Fernandes(1), Farah de Castro Gama(1), \\ Marcos Botton ${ }^{(2)}$ e Andréa Nunes Moreira de Carvalho(3)
}

\begin{abstract}
(1)Embrapa Semiárido, BR-428, Km 152, Zona Rural, no 23, CEP 56302-970 Petrolina, PE, Brasil. E-mail: eudes.oliveira@embrapa.br, herlandia_fernandes@hotmail.com, farah.gama@embrapa.br (2)Embrapa Uva e Vinho, Rua Livramento, no 515, CEP 95700-000 Bento Gonçalves, RS, Brasil. E-mail: marcos.botton@embrapa.br ${ }^{(3)}$ Instituto Federal do Sertão Pernambucano, BR-407, Km 08, Jardim São Paulo, CEP 56314-520 Petrolina, PE, Brasil. E-mail: anmcarvalho@yahoo.com.br
\end{abstract}

Resumo - O objetivo deste trabalho foi avaliar a eficiência da técnica de confusão sexual, com o feromônio sexual sintético composto (Z)-11-hexadecenal (Z11-16: Ald) a 1,8\% e (Z)-13-octadecenal (Z13-18: Ald) a $1,8 \%$, no controle da população de traça-dos-cachos, Cryptoblabes gnidiella (Lepidoptera: Pyralidae), em cultivares de videiras ( Vitis vinifera) destinadas à produção de vinhos. Os experimentos foram realizados em duas localidades na região do Vale do São Francisco, em áreas comerciais de produção de uva para processamento, com as cultivares Cabernet Sauvignon, Petit Verdot, Tempranillo e Chenin Blanc. Os tratamentos avaliados foram os seguintes: confusão sexual com uso de liberadores Splat ("specialized pheromone and lure application technology"), aplicados com pistola manual a 500 pontos por hectare ( $2 \mathrm{~g}$ por ponto); e testemunha sem aplicação. O efeito da técnica sobre os adultos de C. gnidiella foi avaliado com uso de armadilha tipo Delta, iscada em um septo com $2 \mathrm{~g}$ de feromônio sintético da mesma formulação. Os danos nos cachos foram avaliados à colheita. $\mathrm{O}$ uso da técnica de confusão sexual reduziu a captura de adultos de $C$. gnidiella em mais de $59 \%$ na 'Tempranillo', 68\% na 'Chenin Blanc', 80\% na 'Cabernet Sauvignon' e 97\% na 'Petit Verdot'. À época da colheita, os danos nos frutos foram reduzidos de 65 a 100\% nas áreas tratadas. O feromônio sexual sintético é eficaz para reduzir o acasalamento de $C$. gnidiella em vinhedos, com o uso da técnica de confusão sexual.

Termos para indexação: Vitis vinifera, controle comportamental, feromônio sexual, manejo integrado de pragas, traça-dos-cachos, uva de vinho.

\section{Using the technique of mating disruption for Cryptoblabes gnidiella (Lepidoptera: Pyralidae) population management on grapevine}

\begin{abstract}
The objective of this work was to evaluate the efficiency of mating disruption technique, with the synthetic sex pheromone compound (Z)-11-hexadecenal (Z11-16: Ald) at 1.8\% and (Z)-13-octadecenal (Z13-18: Ald) at 1.8\%, for controlling the honeydew moth, Cryptoblabes gnidiella (Lepidoptera: Pyralidae), population in grape (Vitis vinifera) cultivars destined to wine production. The experiments were carried out in two locations in the region of São Francisco Valley, Brazil, in commercial production areas of grapes used for processing, with the cultivars Cabernet Sauvignon, Petit Verdot, Tempranillo, and Chenin Blanc. The evaluated treatments were: mating disruption using Splat (specialized pheromone and lure application technology) releasers, applied at 500 points per hectare ( $2 \mathrm{~g}$ per point); and a control without application. The effect of the technique was evaluated on adults of $C$. gnidiella using Delta type trap baited with $2 \mathrm{~g}$ of synthetic pheromone of the same formulation. The damage on grape clusters was evaluated at harvest. The use of mating disruption technique reduced the capture of adult C. gnidiella in more than $59 \%$ in 'Tempranillo', $68 \%$ in 'Chenin Blanc', 80\% in 'Cabernet Sauvignon', and 97\% in 'Petit Verdot'. At harvest, fruit damage was reduced from 65 to $100 \%$ in the treated areas. The synthetic sex pheromone is effective in reducing C. gnidiella mating in vineyards, using the mating disruption technique.
\end{abstract}

Index terms: Vitis vinifera, behavioral control, sexual pheromone, integrated pest management, honeydew moth, wine grapes.

\section{Introdução}

A traça-dos-cachos da videira, Cryptoblabes gnidiella (Millière, 1867) (Lepidoptera: Pyralidae), é uma espécie polífaga (Singh \& Singh, 1997), nativa da região Mediterrânea (Scatoni \& Bentancourt, 1983). Ela está amplamente dispersa na bacia do Mediterrâneo e presente também na Malásia, Nova 
Zelândia, nas ilhas havaianas, principalmente na África e na Ásia, e em muitas regiões tropicais e subtropicais das Américas (Lucchi et al., 2011). No Brasil, no Rio Grande do Sul e na região do Vale do São Francisco, em Pernambuco, esta praga tem-se destacado por causar danos significativos à cultura da videira (Ringenberg et al., 2006), principalmente às cultivares destinadas à elaboração de vinho (Vitis vinifera $\mathrm{L}$.).

As lagartas de C. gnidiella abrigam-se nos cachos com frutos ainda verdes, alimentam-se da casca do engaço e causam o murchamento e a queda das bagas (Ringenberg et al., 2006). Outros prejuízos ocorrem quando o ataque acontece próximo à colheita e a espécie causa o rompimento das bagas e a liberação do suco, o que possibilita a proliferação de bactérias causadoras da podridão ácida, com reflexos na qualidade dos vinhos e dos frutos para comércio in natura (Lucchi et al., 2011).

As maiores dificuldades para o controle da traça-dos-cachos, em cultivares para produção de vinho, referem-se ao hábito da praga em se abrigar no interior dos cachos. Além disso, a ausência da prática de raleio resulta em cachos mais compactos, o que deixa a praga protegida de inimigos naturais e da ação de inseticidas. Assim, novas estratégias de controle têm sido pesquisadas. $\mathrm{O}$ uso de feromônios sexuais é uma estratégia que apresenta grande potencial no controle de pragas agrícolas (Zarbin et al., 2009). Após a identificação do feromônio sexual de $C$. gnidiella por Bjostad et al. (1981), alguns estudos realizados com seu emprego foram realizados para o monitoramento da espécie. Na Itália, Bagnoli \& Lucchi (2001) monitoraram adultos de $C$. gnidiella em videira. No Uruguai, Vidart et al. (2013) utilizaram armadilhas com o feromônio sexual sintético, para monitorar populações da praga em diferentes cultivares de videira. No Brasil, Bisotto-de-Oliveira et al. (2007) monitoraram a ocorrência de adultos de $C$. gnidiella em diferentes estágios fenológicos da videira, com uso desse feromônio sexual.

Além do uso para fim de monitoramento, é possível o emprego desses feromônios para o controle da praga. O uso da técnica de confusão sexual consiste em saturar o ambiente com o odor da fêmea, o que interfere ou bloqueia a comunicação olfativa entre parceiros sexuais. A liberação do feromônio sexual tem a finalidade de confundir o macho e dificultar o encontro e o acasalamento dos insetos, o que dificultaria a reprodução da espécie e resultaria em declínio populacional (Cardé \& Minks, 1995).

O objetivo deste trabalho foi avaliar a eficiência da técnica de confusão sexual, com o uso do feromônio sexual composto (Z)-11-hexadecenal (Z11-16: Ald) a 1,8\%, e (Z)-13-octadecenal (Z13-18: Ald) a 1,8\%, para o controle da traça-dos-cachos em cultivares de videiras destinadas à produção de vinhos.

\section{Material e Métodos}

O trabalho foi realizado em áreas de produção comercial de uva para processamento, na região do Submédio do Vale do São Francisco, implantadas com o sistema de espaldeira e mantidas com manejo convencional. O primeiro experimento foi instalado no segundo semestre de 2010, em vinhedo comercial ( $9^{\circ} 04^{\prime} 46^{\prime \prime} \mathrm{S}, 40^{\circ} 08^{\prime} 20^{\prime \prime} \mathrm{W}$, a $365 \mathrm{~m}$ de altitude), no Município de Lagoa Grande, PE, com as cultivares Cabernet Sauvignon e Petit Verdot, plantadas em espaçamento $3,2 \times 1,0 \mathrm{~m}$ e 3,0x2,0 m, respectivamente. $\mathrm{O}$ segundo experimento foi instalado em vinhedo comercial $\left(9^{\circ} 14^{\prime} 37^{\prime \prime} \mathrm{S}, 40^{\circ} 53^{\prime} 44^{\prime \prime} \mathrm{W}\right.$; a $457 \mathrm{~m}$ de altitude) no Município de Casa Nova, BA, com a cultivar Tempranillo no segundo semestre de 2010, em espaçamento de 3,0x1,0 m e, no primeiro semestre de 2012 com a cultivar Chenin Blanc, no espaçamento de $2,2 \times 1,0 \mathrm{~m}$.

A técnica de confusão sexual foi aplicada com uso de liberadores Splat ("specialized pheromone and lure application technology"), com (Z)-11-hexadecenal (Z11-16: Ald) a 1,8\% e (Z)-13-octadecenal (Z13-18: Ald) a 1,8\% (Isca Tecnologias Ltda., Ijuí, RS, Brasil). O produto foi aplicado manualmente, a 500 pontos ha- ${ }^{-1}$, nos mourões de sustentação do parreiral. Para tal, foi empregado um aplicador constituído de uma pistola adaptada com dosador, tendo-se utilizado $2 \mathrm{~g}$ por ponto, à dose de um $1 \mathrm{~kg} \mathrm{ha}^{-1}$.

Os experimentos foram realizados em quatro áreas comerciais, com quatro diferentes cultivares. Para cada uma dessas áreas e cultivares, foram avaliados dois tratamentos, um sem aplicação de feromônio (testemunha) e outro com aplicação do feromônio sexual sintético. As parcelas foram de 3 ha em cada área e cultivar. As áreas utilizadas permaneceram com o manejo convencional do produtor, ou seja, recebiam os mesmos tratos culturais de adubação, irrigação, poda etc; porém, sem aplicação de inseticidas para o manejo da praga. 
O efeito do tratamento foi avaliado pelo registro da flutuação populacional de adultos de $C$. gnidiella ao longo da safra. Em cada área e em cada tratamento, foram usadas três armadilhas modelo Delta de cor branca, iscadas com um septo com feromônio sexual sintético de C. gnidiella ( $2 \mathrm{~g})$. Cada armadilha foi posicionada em pontos equidistantes e mantida à altura aproximada de 1,8 $\mathrm{m}$ do solo. As armadilhas foram monitoradas semanalmente, a partir do sétimo dia da aplicação do feromônio, tendo-se quantificado os machos capturados. A substituição da base adesiva foi feita a cada 30 dias, com a troca do septo de borracha com feromônio (Bisotto-de-Oliveira et al., 2007).

Para avaliar o dano causado pelo inseto à colheita, foram avaliados 500 cachos de uva de cada tratamento, com cinco repetições de 100 cachos cada. Nesta avaliação, os cachos foram escolhidos aleatoriamente e avaliados em campo, de forma a abranger toda a área. Para cada cacho amostrado, foi realizada a inspeção e foi registrada a presença de ovos e lagartas, bem como a de danos causados pelo inseto.

Nos diferentes tratamentos, avaliou-se graficamente a flutuação populacional de adultos, pela plotagem do número médio de machos por armadilha por semana, em função do tempo. O número total médio de insetos capturados nas três armadilhas, em cada unidade experimental, foi comparado em todo o período experimental.

Para comparar o número de machos por tratamento, procedeu-se à análise de variância pelo modelo linear generalizado (GLM) (Crawley, 2007), com distribuição de erros Poisson. A variável resposta (y) correspondeu ao número de insetos capturados por armadilha, e a variável explicativa (x), aos tratamentos (confusão sexual e testemunha). Para a avaliação de danos, utilizou-se como variável resposta (y) o número de cachos com ovos e lagartas ou com danos e, como variável explicativa (x), os tratamentos (confusão sexual e testemunha). As análises estatísticas foram realizadas com o software R (R Development Core Team, 2010). A percentagem de redução de danos foi calculada pela fórmula de Abbott (1925).

\section{Resultados e Discussão}

A aplicação do feromônio sexual sintético reduziu significativamente o número médio de machos capturados nas armadilhas, em Lagoa Grande, PE, na 'Cabernet Sauvignon', na safra 2010, e na 'Petit Verdot' na safra 2010/2011. Os experimentos realizados em Casa Nova, BA, com a 'Tempranillo', na safra 2010, e 'Chenin Blanc', na safra 2012, tiveram resultados semelhantes (Tabela 1).

$\mathrm{Na}$ 'Cabernet Sauvignon', o pico de captura de machos foi observado no tratamento testemunha, que apresentou média de 179 insetos por armadilha, enquanto na área tratada com feromônio sexual a média foi de 15,6 , o que representou redução de $91 \%$ na captura, pelo uso da técnica de confusão sexual. Em geral, observou-se redução na captura de machos de $80 \%$, no mínimo, na área com essa cultivar, em razão do tratamento (Figura 1). Na 'Petit Verdot', safra 2010/2011, o pico no número médio de machos capturados, registrado em $7 / 2 / 2011$, foi de 300 insetos por armadilha, na testemunha, e de seis insetos na área tratada. Semelhantemente aos resultados obtidos com a 'Cabernet Sauvignon', a área tratada apresentou redução de 97\%, no mínimo, na captura de machos de C. gnidiella, na 'Petit Verdot'.

Para a 'Tempranilllo', na safra 2010, foram registrados dois picos populacionais no tratamento testemunha, em 4/10/2010 e 1/11/2010, com número médio de machos capturados por armadilha de 35,3 e 43,3,

Tabela 1. Efeito da técnica de confusão sexual sobre o número de machos adultos de Cryptoblabes gnidiella, capturados em armadilhas tipo Delta em cultivos comerciais de videira (Vitis vinifera), em diferentes localidades, durante todo o período experimental $^{(1)}$.

\begin{tabular}{|c|c|c|c|c|c|c|c|c|}
\hline \multirow[t]{3}{*}{ Tratamento } & \multicolumn{4}{|c|}{ Lagoa Grande, PE } & \multicolumn{4}{|c|}{ Casa Nova, BA } \\
\hline & \multicolumn{2}{|c|}{ 'Cabernet Sauvignon' } & \multicolumn{2}{|c|}{ 'Petit Verdot' } & \multicolumn{2}{|c|}{ 'Tempranillo' } & \multicolumn{2}{|c|}{ 'Chenin blanc' } \\
\hline & Média $\pm \mathrm{EP}$ & Significância & Média $\pm \mathrm{EP}$ & Significância & Média $\pm E P$ & Significância & Média $\pm \mathrm{EP}$ & Significância \\
\hline Confusão sexual & $4,5 \pm 1,56$ & $\mathrm{~F}=106,44$ & $1,66 \pm 0,58$ & $\mathrm{~F}=110,51$ & $1,6 \pm 0,59$ & $\mathrm{~F}=259,2$ & $10,08 \pm 5,31$ & $F=25,66$ \\
\hline Testemunha & $62,0 \pm 12,41$ & $\mathrm{p}<0,001^{* *}$ & $153,51 \pm 24,76$ & $\mathrm{p}<0,001^{* *}$ & $21,2 \pm 3,30$ & $\mathrm{p}<0,001^{* *}$ & $94,79 \pm 32,31$ & $\mathrm{p}<0,01 * *$ \\
\hline
\end{tabular}

(1)'Cabernet Sauvignon', 14 semanas; 'Petit Verdot', 11 semanas; 'Tempranilho', 12 semanas; e 'Chenin blanc', 8 semanas. *Significativo a $1 \%$ de probabilidade. EP, erro-padrão da média. 
respectivamente. $\mathrm{Na}$ área tratada, a média de captura alcançou, no máximo, 6,7 machos por armadilha, em 6/9/2010. Em geral, observou-se que a confusão sexual reduziu a captura em 59\%, no mínimo, em comparação à testemunha. Em algumas avaliações semanais, houve até mesmo casos de ausência total de captura na área

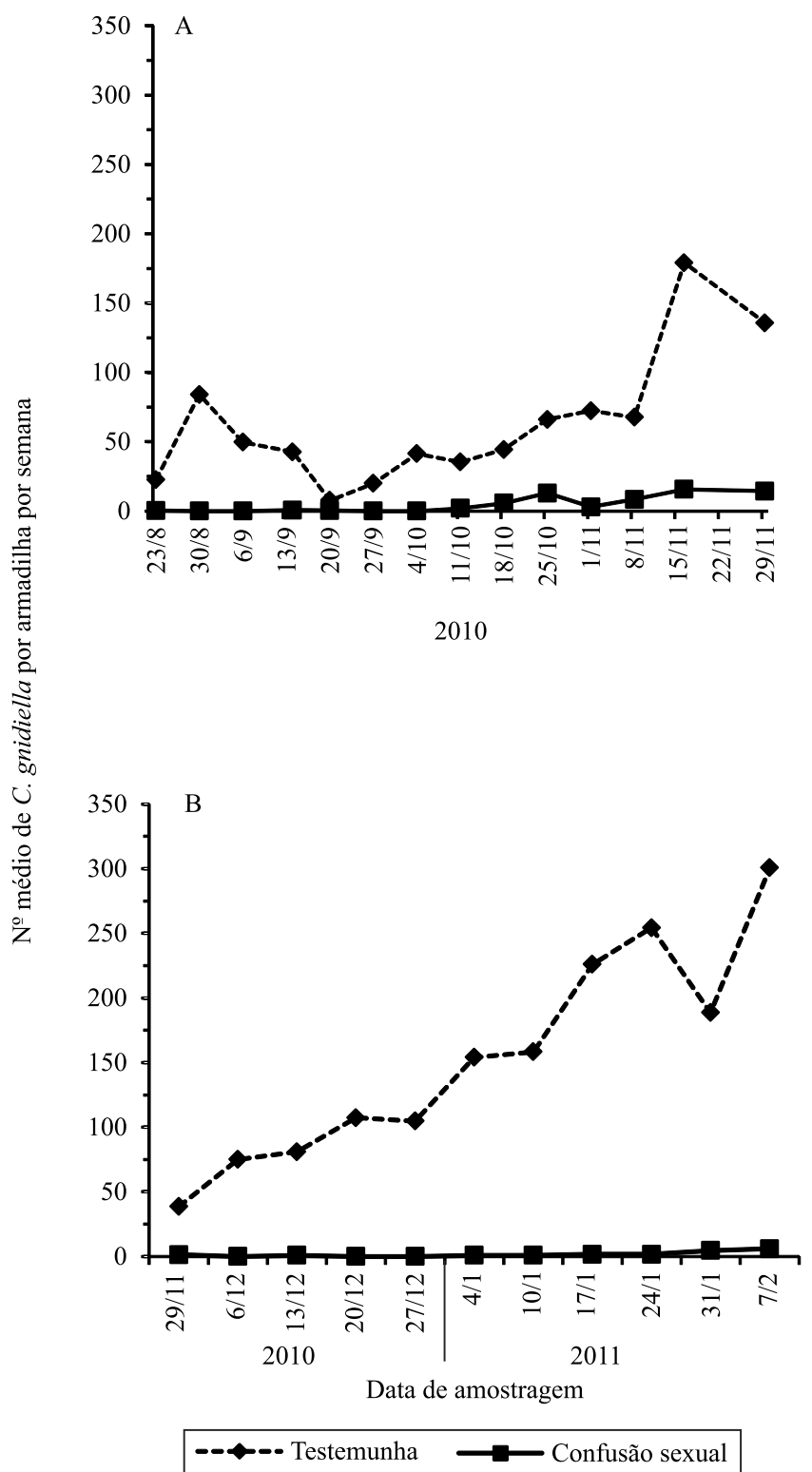

Figura 1. Flutuação populacional de adultos de Cryptobables gnidiella, capturados nas cultivares de uva Cabernet Sauvignon, no ano de 2010 (A), e Petit Verdot, na safra 2010/2011 (B), por armadilha Delta, em áreas com ou sem o uso da técnica de confusão sexual, em Lagoa Grande, PE. tratada (Figura 2). Para a 'Chenin Blanc', na safra 2012, registrou-se em 21/5/2012 o pico de captura de 221,3 insetos por armadilha na testemunha e, na mesma data, de 42,6 insetos por armadilha na área tratada, o que representou redução de $81 \%$ na captura na área tratada. Em todas as avaliações realizadas, observou-

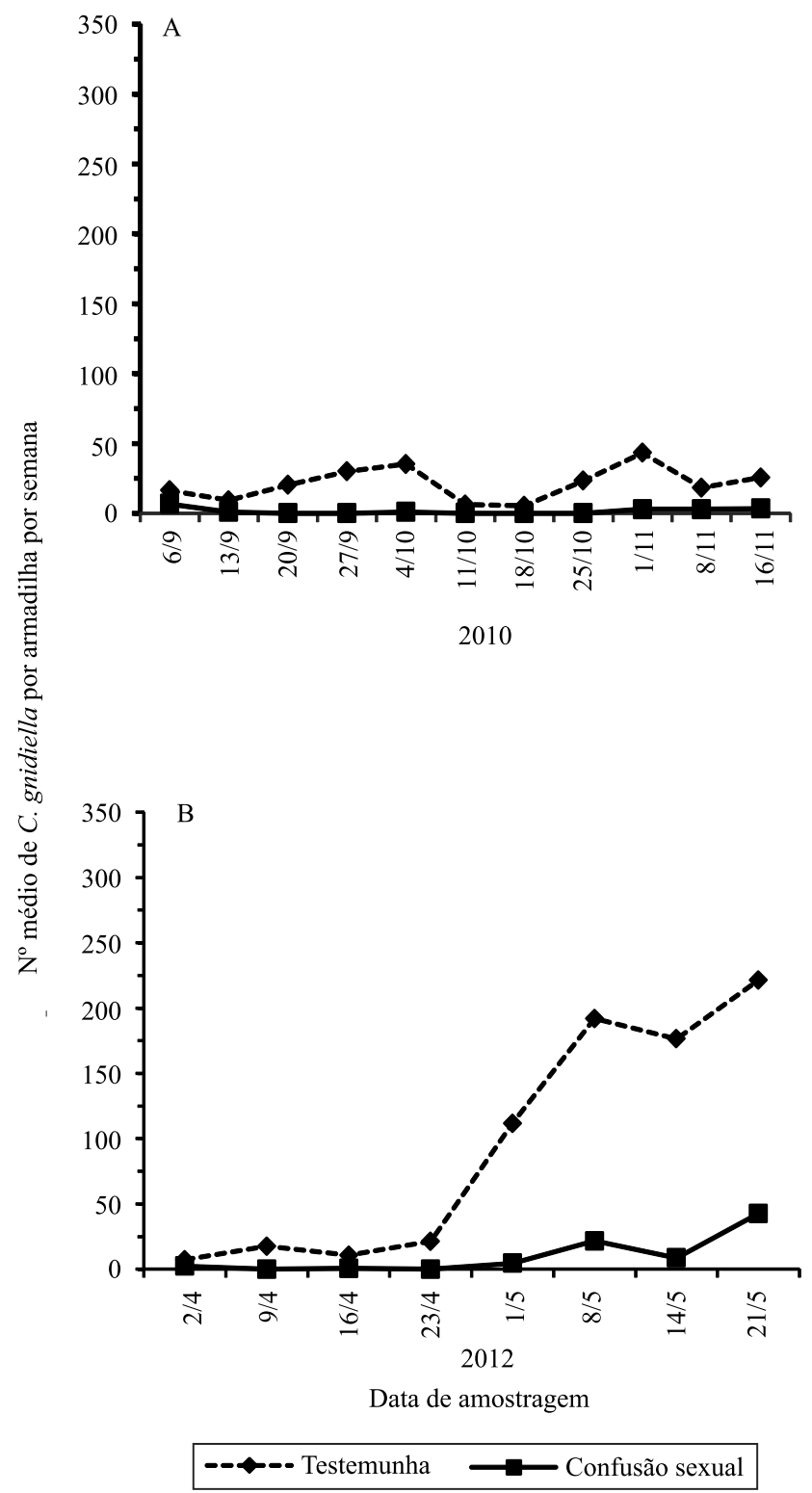

Figura 2. Flutuação populacional de adultos de Cryptobables gnidiella, capturados por armadilha Delta nas cultivares de uva Tempranillo, no ano de 2010 (A), e Chenin Blanc, no ano de 2012 (B), em áreas com ou sem o uso da técnica de confusão sexual, em Casa Nova, BA. 
se redução de 68 a $100 \%$ na captura de machos de C. gnidiella.

De acordo com esse resultados, a técnica de confusão sexual apresentou desempenho promissor no controle de $C$. gnidiella, em ambas as localidades avaliadas, independentemente da cultivar utilizada.

Quanto à incidência de danos nos cachos, determinada em avaliações realizadas no momento da colheita, observou-se que as áreas não tratadas tiveram até 5,2\% de cachos atacados na 'Cabernet Sauvignon' e de $0,4 \%$ na 'Chenin Blanc'. Nas áreas tratadas, essa percentagem baixou para 1,8 e $0 \%$, para as mesmas cultivares. Para 'Petit Verdot' e 'Tempranillo', independentemente do tratamento, não se observou presença de danos nos cachos. Em qualquer amostragem em que se detectou presença de danos, a intensidade de ataque da praga, expressa pela percentagem de cachos atacados, diferiu significativamente entre os tratamentos. Em geral, houve redução de, no mínimo, $65 \%$ na ocorrência de danos causados por C. gnidiella aos cachos de videira, pelo emprego da técnica de confusão sexual (Tabela 2).

Os danos observados nos cachos, no momento da colheita, não chegam a indicar falha na eficiência da técnica, pois, segundo Cardé \& Minks (1995), a presença de danos nos cachos pode ocorrer também pela migração de fêmeas acasaladas de áreas adjacentes. Além disso, em algumas situações, a técnica pode não apresentar a eficência esperada, conforme relatado por: Ambrogi et al. (2006), sobre Leucoptera coffeella (Guérin-Meneville) (Lepidoptera: Lyonetiidae), na cultura do café; e por Higbee \& Burks (2008), sobre Amyelois transitella (Walker) (Lepidoptera: Pyralidae) em pistache.

De acordo com Sanders (1989), muitos fatores podem contribuir para o insucesso da técnica de confusão sexual, como alta densidade populacional, disseminação baixa ou irregular do feromônio, ocorrência de ventos intensos, alta temperatura e imigração de fêmeas acasaladas de áreas não tratadas.
Além desses fatores, outra possível dificuldade da técnica refere-se à instabilidade do feromônio natural, pois os aldeídos são instáveis em condições de campo, em razão da rápida oxidação e polimerização (Ujváry et al., 1993). Assim, Sellanes et al. (2010) estudaram as respostas eletrofisiológicas e comportamentais de machos de $C$. gnidiella às formas análogas de (Z)-11-hexadecenal e (Z)-13-octadecenal, dada a importância destes análogos estruturais mais estáveis, para aumentar a eficácia da técnica e potencializar seu uso no manejo de pragas.

Entretanto, assim como os resultados aqui apresentados, outros trabalhos também têm mostrado resultados satisfatórios, quanto à eficiência da técnica de confusão sexual, em diversas pragas agrícolas, tais como: Grapholita molesta (Busck) (Lepidoptera: Tortricidae), nas culturas de pêssego (Härter et al., 2010) e maçã (Pastori, 2007; Monteiro et al., 2013); Bonagota salubricola (Meyrick) (Lepidoptera: Tortricidae) (Pastori, 2007) e Cydia pomonella (L.) (Lepidoptera: Tortricidae) (Ashraf et al., 2007), em maçã; Lobesia botrana (Lepidoptera : Tortricidae), em videira (Vassiliou, 2009); Zeuzera pyrina (L.) (Lepidoptera: Cossidae), em oliveiras (Hegazi et al., 2010); Pectinophora gossypiella (Saunders) (Lepidoptera: Gelechiidae), em algodão (Unlu \& Mezreli, 2011); e Tuta absoluta (Meyrick) (Lepidoptera: Gelechiidae), na cultura do tomate (Cocco et al., 2013).

As ações de controle com uso de semioquímicos contribuem para um modelo adequado para a agricultura do futuro, pois apresentam alta especificidade e não há depósito significativo de elementos químicos no meio ambiente ou no alimento produzido (Zarbin et al., 2009). Assim, os resultados obtidos no presente estudo mostram a eficiência da técnica em reduzir os encontros entre machos e fêmeas, que constitui, assim, uma estratégia adequada para o controle sustentável de C. gnidiella em videiras.

Tabela 2. Efeito da técnica de confusão sexual sobre a percentagem de cachos danificados por Cryptoblabes gnidiella, em cultivos comerciais de videira (Vitis vinifera), em diferentes localidades, durante a colheita.

\begin{tabular}{|c|c|c|c|c|c|c|c|c|}
\hline \multirow[t]{3}{*}{ Tratamento } & \multicolumn{4}{|c|}{ Lagoa Grande, PE } & \multicolumn{4}{|c|}{ Casa Nova, BA } \\
\hline & \multicolumn{2}{|c|}{ 'Cabernet Sauvignon' } & \multicolumn{2}{|c|}{ 'Petit Verdot' } & \multicolumn{2}{|c|}{ 'Tempranillo' } & \multicolumn{2}{|c|}{ 'Chenin blanc' } \\
\hline & Média $\pm \mathrm{EP}$ & Significância & Média $\pm \mathrm{EP}$ & Significância & Média $\pm E P$ & Significância & Média \pm EP & Significância \\
\hline Confusão sexual & $1,8 \pm 0,05$ & $\mathrm{~F}=476,37$ & 0,0 & - & 0,0 & - & 0,0 & $\mathrm{~F}=143,12$ \\
\hline Testemunha & $5,2 \pm 0,13$ & $\mathrm{p}<0,001 * *$ & 0,0 & - & 0,0 & - & $0,4 \pm 0,03$ & $\mathrm{p}<0,01 * *$ \\
\hline
\end{tabular}

\footnotetext{
**Significativo a $1 \%$ de probabilidade. EP, erro-padrão da média.
} 


\section{Conclusões}

1. Os feromônios sexuais sintéticos(Z)-11-hexadecenal (Z11-16: Ald) a 1,8\% e (Z)-13-octadecenal (Z13-18: Ald) a 1,8\% são eficazes para a redução do acasalamento de Cryptoblabes gnidiella, em cultivos comerciais de cultivares de uva destinadas à vinificação.

2. A técnica de confusão sexual reduz o dano causado por $C$. gnidiella a cachos de videira.

\section{Agradecimentos}

À Fundação de Amparo à Ciência e Tecnologia do Estado de Pernambuco (Facepe), pelo apoio financeiro; e ao Conselho Nacional de Desenvolvimento Científico e Tecnológico (CNPq), pela concessão de bolsa.

\section{Referências}

ABBOTT, W.S. A method of computing the effectiveness of an insecticide. Journal of Economic Entomology, v.18, p.265-267, 1925

AMBROGI, B.G.; LIMA, E.R.; SOUSA-SOUTO, L. Efficacy of mating disruption for control of the coffee leaf miner Leucoptera coffeella (Guérin-Méneville) (Lepidoptera: Lyonetiidae). Bioassay, v.1, p.1-5, 2006.

ASHRAF, M.; ASIF, M.; ADREES, M.; HANIF, W.; ISHTIAQ, CH.M. Application of mating disruption approach to control codling moth (Cydia pomonella L.) damage to apple crops in Azad Kashmir, Pakistan. Pakistan Journal of Biological Sciences, v.10, p.1728-1732, 2007. DOI: 10.3923/pjbs.2007.1728.1732.

BAGNOLI, B.; LUCCHI, A. Bionomics of Cryptoblabes gnidiella (Millière) (Pyralidae, Phycitinae) in Tuscan vineyards. IOBC-WPRS Bulletin, v.24, p.79-83, 2001.

BISOTTO-DE-OLIVEIRA, R.; REDAELLI, L.R; SANT'ANA, J.; COVER, C.; BOTTON, M. Ocorrência de Cryptoblabes gnidiella (Millière) (Lepidoptera: Pyralidae) relacionada à fenologia da videira em Bento Gonçalves, RS. Neotropical Entomology, v.36, p.555-559, 2007. DOI: 10.1590/ S1519-566X2007000400013.

BJOSTAD, L.B.; GUREVITZ, E.; GOTHILF, S.; ROELOFS, W.L. Sex attractant for the honeydew moth, Cryptoblabes gnidiella. Phytoparasitica, v.9, p.95-99, 1981. DOI: 10.1007/BF03158451.

CARDÉ, R.T.; MINKS, A.K. Insect pheromone research: new directions. New York: Chapman \& Hall, 1995. 648p.

COCCO, A.; DELIPERI, S.; DELRIO, G. Control of Tuta absoluta (Meyrick) (Lepidoptera: Gelechiidae) in greenhouse tomato crops using the mating disruption technique. Journal of Applied Entomology, v.137, p.16-28, 2013. DOI: 10.1111/j.1439-0418.20 12.01735.x

CRAWLEY, M.J. Statistics: an introduction using R. Oxford: Wiley, 2007. 761p.
HÄRTER, W. da R.; GRÜTZMACHER, A.D.; NAVA, D.E.; GONÇALVES, R. da S.; BOTTON, M. Isca tóxica e disrupção sexual no controle da mosca-da-fruta sul-americana e da mariposa-oriental em pessegueiro. Pesquisa Agropecuária Brasileira, v.45, p.229-235, 2010. DOI: 10.1590/S0100-204X2010000300001.

HEGAZI, E.M.; KHAFAGI, W.E.; KONSTANTOPOULOU, M.A.; SCHLYTER, F.; RAPTOPOULOS, D.; SHWEIL, S.; ABD EL-RAHMAN, S.; ATWA, A.; ALI, S.E.; TAWFIK, H. Suppression of leopard moth (Lepidoptera: Cossidae) populations in olive trees in Egypt through mating disruption. Journal of Economic Entomology, v.103, p.1621-1627, 2010. DOI: 10.1603/EC09435.

HIGBEE, B.S; BURKS, C.S. Effects of mating disruption treatments on navel orangeworm (Lepidoptera: Pyralidae) sexual communication and damage in almonds and pistachios. Journal of Economic Entomology, v.101, p.1633-1642, 2008. DOI: 10.1603/0022-0493(2008)101[1633:EOMDTO]2.0.CO;2.

LUCCHI, A.; BOTTON, M.; BAGNOLI, B. Tignola rigata su vite da tenere sotto controllo. L' Informatore Agrario, v.31, p.65-70, 2011.

MONTEIRO, L.B.; SOUZA, A; ARGENTON, J. Mating disruption with low density diffusers for the management of oriental fruit moths (Lepidoptera: Tortricidae) in apple orchards under subtropical climate in Southern Brazil. Revista Brasileira de Fruticultura, v.35, p.1007-1016, 2013. DOI: 10.1590/ S0100-29452013000400011.

PASTORI, P.L. Bioecologia de Trichogramma pretiosum (Riley, 1879) (Hymenoptera: Trichogrammatidae) e controle integrado de Bonagota salubricola (Meyrick, 1937) e Grapholita molesta (Busck, 1916) (Lepidoptera: Tortricidae) com feromônios sexuais na cultura da macieira. 2007. 151p. Dissertação (Mestrado) - Universidade Federal do Paraná, Paraná.

R DEVELOPMENT CORE TEAM. The R project for statistical computing. Version 2.12.0. Vienna: R Development Core Team, 2010. Disponível em: <http://www.R-project.org/>. Acesso em: 08 set. 2013.

RINGENBERG, R.; BOTTON, M.; GARCIA, M.S.; AMORIM, F.M.; HAJI, F.N. A traça-dos-cachos da videira. Revista Cultivar HF, v.35, p.31-33, 2006.

SANDERS, C.J. The further understanding of pheromones: biological and chemical research for the future. In: JUTSUM, A.R.; GORDON, R.F.S. (Ed.). Insect pheromones in plant protection. New York: John Wiley, 1989. p.325-351.

SCATONI, I.B.; BENTANCOURT, C.M. Cryptoblabes gnidiella (Millière): una nueva lagarta de los racimos en los viñedos de nuestro país. Revista de la Asociación de Ingenieros Agrónomos, v.1, p.266-268, 1983.

SELLANES, C.; ROSSINI, C.; GONZÁLEZ, A. Formate analogs as antagonists of the sex pheromone of the honeydew moth, Cryptoblabes gnidiella: electrophysiological, behavioral and field evidence. Journal of Chemical Ecology, v.36, p.1234-1240, 2010. DOI: $10.1007 / \mathrm{s} 10886-010-9861-8$.

SINGH, Y.P.; SINGH, D.K. Host plants, extent of damage and seasonal abundance of earhead caterpillar, Cryptoblabes gnidiella 
Miller. Advances in Agricultural Research in India, v.7, p.133-137, 1997.

UJVÁRY, I.; DICKENS, J.C.; KAMM, J.A.; MCDONOUGH, L.M. Natural product analogs: stable mimics of aldehyde pheromones. Archives of Insect Biochemistry and Physiology, v.22, p.393-411, 1993. DOI: 10.1002/arch.940220308.

UNLU, L.; MEZRELI, E. Control of the pink bollworm Pectinophora gossypiella by the mating disruption technique on cotton in a semi-arid region of Turkey. Phytoparasitica, v.39, p.19-25, 2011. DOI: 10.1007/s12600-010-0131-7.

VASSILIOU, V.A. Control of Lobesia botrana (Lepidoptera: Tortricidae) in vineyards in Cyprus using the mating disruption technique. Crop Protection, v.28, p.145-150, 2009. DOI: 10.1016/j.cropro.2008.09.013.

VIDART, M.V.; MUJICA, M.V.; CALVO, M.V.; DUARTE, F.; BENTANCOURT, C.M.; FRANCO, J.; SCATONI, I.B. Relationship between male moths of Cryptoblabes gnidiella (Millière) (Lepidoptera: Pyralidae) caught in sex pheromone traps and cumulative degree-days in vineyards in southern Uruguay. SpringerPlus, v.2, p.1-8, 2013. DOI: 10.1186/2193-1801-2-258.

ZARBIN, P.H.G.; RODRIGUES, M.A.C.M.; LIMA, E.R. Feromônios de insetos: tecnologia e desafios para uma agricultura competitiva no Brasil. Química Nova, v.32, p.722-731, 2009. DOI: $10.1590 /$ S0100-40422009000300016.

Recebido em 23 de abril de 2014 e aprovado em 23 de outubro de 2014 